\title{
Photoelectron circular dichroism of chiral molecules studied with a continuum-state-corrected strong-field approximation
}

\author{
Ingo Dreissigacker* and Manfred Lein \\ Institut für Theoretische Physik and Centre for Quantum Engineering and Space-Time Research (QUEST), \\ Leibniz Universität Hannover, Appelstraße 2, 30167 Hannover, Germany
}

(Received 17 February 2014; published 7 May 2014)

\begin{abstract}
Motivated by recent experiments on circular dichroism in the photoelectron momentum distributions from strong-field ionization of chiral molecules [C. Lux et al., Angew. Chem. Int. Ed. 51, 5001 (2012); C. S. Lehmann et al., J. Chem. Phys. 139, 234307 (2013)], we investigate the origin of this effect theoretically. We show that it is not possible to describe photoelectron circular dichroism with the commonly used strong-field approximation due to its plane-wave nature. We therefore apply the Born approximation to the scattering state and use this as a continuum-state correction in the strong-field approximation. We obtain electron distributions for the molecules camphor and fenchone. In order to gain physical insight into the process, we study the contributions of individual molecular orientations.
\end{abstract}

DOI: 10.1103/PhysRevA.89.053406

PACS number(s): $33.80 . \mathrm{Rv}, 33.80 . \mathrm{Wz}$

\section{INTRODUCTION}

The response of atoms and small molecules to strong, ultrafast laser pulses has been studied extensively over the last years. Impressive progress has been made in the theoretical understanding of phenomena such as above-threshold ionization and high-harmonic generation. This is not only due to the ever-increasing power of computing resources which are used to improve approximations for the quantum mechanical treatment of systems with growing complexity, but also due to the great success of theories such as the strong-field approximation (SFA) [1-5]. While there is still a great interest in the details of strong-field ionization of small systems [6,7], ionization of larger molecules receives increasing attention [8], since the photoelectron angular distributions (PADs) carry important structural information on the molecules. PADs of polyatomic molecules have been calculated successfully within the framework of the SFA [9-11], and can nowadays be efficiently measured using velocity-map imaging [12,13]. For example, velocity-map imaging has been used to track molecular rotation on a subpicosecond time scale [14]. Although PAD measurements often seek to measure molecular-frame PADs [15-17], also PADs of randomly oriented molecules can show interesting features, in particular when the molecules are chiral. One-photon ionization of chiral molecules with a circularly polarized XUV beam can lead to a forwardbackward asymmetry in the photoelectron emission [18,19] with respect to the propagation direction of the light beam. This phenomenon is known as photoelectron circular dichroism (PECD). The asymmetry changes its sign upon changing the enantiomer or the rotation direction of the light field. Thus, the effect does not occur in racemic samples of chiral molecules, i.e., 1:1 mixtures of different enantiomers. A similar behavior is familiar from the conventional dichroism effect in the photoabsorption cross section. However, unlike the conventional dichroism, where the relative difference in the photoabsorption of the enantiomers is small and relies

\footnotetext{
*ingo.dreissigacker@itp.uni-hannover.de
}

on an asymmetry in the magnetic dipole element, the PECD can be described within the electric-dipole approximation and can reach the order of magnitude of the electron yield itself [18]. Asymmetries of up to $20 \%$ have been measured in the photoionization of randomly oriented chiral molecules with synchrotron radiation [20-23]. This makes the PECD a promising tool in the research of chiral molecules, e.g., for chiral recognition in the gas phase. Such applications can have a great impact on a plethora of research areas, including even biological and pharmaceutical research, since many chemical processes in living beings are chirality dependent. For example, the homochirality of life, i.e., the fact that all amino acids in living beings are of $L$ type and that the ribose sugars of nucleic acids are of $D$ type, has yet to be understood [24]. Deeper investigation of the PECD may shine light on this puzzle [25].

Recently, it has been experimentally discovered that the PECD does not only occur in single-photon ionization by XUV radiation, but also in few-photon transitions from strong UV laser pulses [26,27]. In these experiments, a difference of up to about $15 \%$ between the forward and backward direction has been found for the organic molecules camphor and fenchone. The possibility to perform such an experiment with a table-top laser system instead of synchrotron radiation is a major step forward to using the PECD in applications. In contrast to the single-photon case, which is already understood and where usable predictions are possible [28,29], the multiphoton case is much more difficult [27].

In this article, we present a theoretical study that computes the PECD for chiral molecules in multiphoton ionization. Despite the complexity of the problem, our approach does not require any fitting parameters and is nonperturbative in the sense that it can treat ionization processes with an arbitrarily high number of photons. The article is split into three parts. In the first part, we demonstrate that the PECD effect requires a non-plane-wave treatment of the electronic continuum by proving that the SFA yields vanishing asymmetry between forward and backward emission of photoelectrons, i.e., the SFA fails at describing PECD. Therefore, a more sophisticated approximation is necessary, which at the same time keeps the 
numerical effort acceptable. Due to the required orientation averaging and the lack of symmetry in the system, this is not a simple task. In the second part of this paper, we develop an extension of the SFA, in which we use the Born approximation known from scattering theory to obtain a correction to the plane-wave continuum states. With this technique, we compute the PECD effect for pure enantiomeric gases of the molecules camphor and fenchone for three-, four-, and five-photon ionization induced by a circularly polarized UV field. In the last part, we use the same method to determine the dichroism of individual molecular orientations of camphor and fenchone. In this way, we investigate the contributions of these orientations to the total PECD effect.

\section{PECD IN THE STRONG-FIELD APPROXIMATION}

We assume the laser field $\mathbf{E}(t)$ to be circularly polarized in the $(x, y)$ plane of the laboratory coordinate system, i.e., $E_{z}(t)=0$. The PECD effect in this situation would manifest itself in an asymmetry of the momentum distribution when comparing forward $\left(k_{z}>0\right)$ and backward $\left(k_{z}<0\right)$ emission of photoelectrons from a sample of randomly oriented molecules. A mirror transformation of the system at the polarization plane does not change the sense of electric field rotation as a function of time but changes all molecules into their enantiomers. In the electric-dipole approximation, changing the enantiomer therefore implies that the sign of asymmetry is inverted as well. If we consider only sufficiently long multicycle pulses, the photoelectron distributions from randomly oriented molecules are cylindrically symmetric about the $z$ axis. In this case, due to the random orientation, a preferred direction is defined solely by the propagation direction of the laser field. Rotating the propagation direction of the field by $180^{\circ}$ must therefore rotate also the electron distribution by $180^{\circ}$. From the viewpoint of a single molecule, this is equivalent to switching from left- to right-circular polarization in the electric-dipole approximation. Thus, the sign of the asymmetry changes its sign upon switching the sense of polarization. The SFA momentum distribution after strong-field ionization, also known as Keldysh-Faisal-Reiss momentum distribution [1-3], averaged over all orientations, reads (atomic units and length gauge are used throughout this paper)

$$
w^{\mathrm{SFA}}(\mathbf{k})=\int d^{3} \mathcal{R}\left|-i \int_{-\infty}^{\infty} d t\left\langle\mathbf{k}+\mathbf{A}(t)|\mathbf{r} \cdot \mathbf{E}(t)| \psi_{0}^{\mathcal{R}}\right\rangle e^{i S(t)}\right|^{2} .
$$

In this equation, $\mathbf{k}$ is the electron momentum defined in the laboratory frame, $\psi_{0}^{\mathcal{R}}$ is the rotated orbital from which ionization takes place, and $\mathbf{A}(t)=-\int^{t} d t^{\prime} \mathbf{E}\left(t^{\prime}\right)$ corresponds to the electric field $\mathbf{E}(t)$ of the laser pulse. The scalar function $S(t)$ is the action

$$
S(t)=I_{p} t+\frac{1}{2} \int^{t} d t^{\prime}\left[\mathbf{k}+\mathbf{A}\left(t^{\prime}\right)\right]^{2}
$$

with the molecular ionization potential $I_{p}$. The bra $\langle\mathbf{k}+\mathbf{A}(t)|$ represents a plane wave with momentum $\mathbf{k}+\mathbf{A}(t)$. The integral over all possible molecular orientations is indicated by the integration $\int d^{3} \mathcal{R}$, where $\mathcal{R}$ is the rotation matrix for an active rotation about the Euler angles $(\eta, \xi, \chi)$ in the $z y z$ convention [30]. Expanding the absolute square in Eq. (1) and rearranging the order of integration leads to the expression

$$
\begin{aligned}
w^{\mathrm{SFA}}(\mathbf{k})= & \frac{1}{(2 \pi)^{3}} \int d t \int d t^{\prime} \int d^{3} r \int d^{3} r^{\prime} e^{-i[\mathbf{k}+\mathbf{A}(t)] \mathbf{r}} \\
& \times[\mathbf{r} \cdot \mathbf{E}(t)] e^{i S(t)} e^{i\left[\mathbf{k}+\mathbf{A}\left(t^{\prime}\right)\right] \mathbf{r}^{\prime}}\left[\mathbf{r}^{\prime} \cdot \mathbf{E}\left(t^{\prime}\right)\right] e^{-i S\left(t^{\prime}\right)} \\
& \times \int d^{3} \mathcal{R} \psi_{0}^{\mathcal{R}}(\mathbf{r}) \psi_{0}^{* \mathcal{R}}\left(\mathbf{r}^{\prime}\right) .
\end{aligned}
$$

To determine the orientation average on the right-hand side of Eq. (3), we use the partial-wave expansion $\psi_{0}(\mathbf{r})=$ $\sum_{l, m} a_{l m}(r) Y_{l m}(\hat{\mathbf{r}})$ of the initial state and the Wigner- $D$-matrix $D_{m^{\prime} m}^{l}(\mathcal{R})$ to rotate the angular momentum eigenstates (see, e.g., Refs. [30,31]):

$$
\psi_{0}^{\mathcal{R}}(\mathbf{r})=\sum_{l=0}^{\infty} \sum_{m, m^{\prime}=-l}^{+l} a_{l m}(r) Y_{l m^{\prime}}(\hat{\mathbf{r}}) D_{m^{\prime} m}^{l}(\mathcal{R})
$$

Here, $\hat{\mathbf{r}}$ denotes the angular coordinates $(\vartheta, \varphi)$ and $r=|\mathbf{r}|$. Using this in conjunction with the orthonormality relation of the Wigner- $D$-matrix, we have

$$
\begin{aligned}
& \int d^{3} \mathcal{R} \psi_{0}^{\mathcal{R}}(\mathbf{r}) \psi_{0}^{* \mathcal{R}}\left(\mathbf{r}^{\prime}\right) \\
& =\sum_{l=0}^{\infty} \sum_{m, m^{\prime}=-l}^{+l} \frac{8 \pi^{2}}{2 l+1} a_{l m}(r) a_{l m}^{*}\left(r^{\prime}\right) Y_{l m^{\prime}}(\hat{\mathbf{r}}) Y_{l m^{\prime}}^{*}\left(\hat{\mathbf{r}}^{\prime}\right)
\end{aligned}
$$

Inserting this back into Eq. (3), and considering $\mathbf{E}(t) \perp \mathbf{e}_{3}$ as well as $\mathbf{A}(t) \perp \mathbf{e}_{3}$, we find the following expression for the momentum distribution:

$$
\begin{aligned}
& w^{\mathrm{SFA}}(\mathbf{k})=\frac{1}{\pi} \sum_{l, m, m^{\prime}} \frac{1}{2 l+1} \int d t d t^{\prime} d^{2} r_{12} d^{2} r_{12}^{\prime} e^{-i[\mathbf{k}+\mathbf{A}(t)] \mathbf{r}_{12}} \\
& \times[\mathbf{r} \cdot \mathbf{E}(t)] e^{i S(t)} e^{i[\mathbf{k}+\mathbf{A}(t)] \mathbf{r}_{12}^{\prime}}\left[\mathbf{r}^{\prime} \cdot \mathbf{E}\left(t^{\prime}\right)\right] e^{-i S\left(t^{\prime}\right)} \\
& \times \int d z d z^{\prime} a_{l m}(r) a_{l m}^{*}\left(r^{\prime}\right) Y_{l m^{\prime}}(\hat{\mathbf{r}}) Y_{l m^{\prime}}^{*}\left(\hat{\mathbf{r}}^{\prime}\right) e^{-i k_{3} z} e^{i k_{3} z^{\prime}} .
\end{aligned}
$$

Here, the subscript label " 12 " denotes coordinates $x, y, x^{\prime}, y^{\prime}$ in the plane of polarization and the subscript label " 3 " the components along the propagation direction of the field. The vanishing PECD can now be shown directly with a symmetry argument regarding Eq. (6): If we compare forward $\left(k_{3}>0\right)$ and backward emission $\left(k_{3}<0\right)$ by changing the sign of $k_{3}$ in Eq. (6), we observe that the substitution $k_{3} \rightarrow-k_{3}$ affects only the exponential functions on the very right of Eq. (6). Here, the change in sign can be undone by substituting $z \rightarrow-z$ and $z^{\prime} \rightarrow-z^{\prime}$. The sign of the $z$ and $z^{\prime}$ coordinates appears besides the exponential functions only in the spherical harmonics $Y_{l m^{\prime}}(\hat{\mathbf{r}}) Y_{l m^{\prime}}^{*}\left(\hat{\mathbf{r}}^{\prime}\right)$. The product of two spherical harmonics with identical quantum numbers is invariant under this transformation. This means that the orientation-averaged momentum distributions in the forward direction and in the backward direction are equal. Consequently, we conclude that any dichroism effect in the PAD calculated with SFA will disappear after orientation averaging, rendering the SFA useless for this particular problem. 
(a)

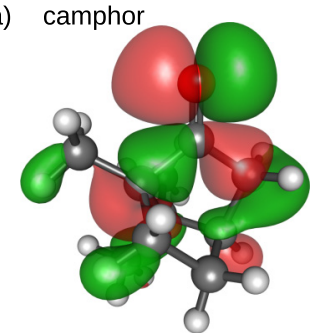

(b) fenchone

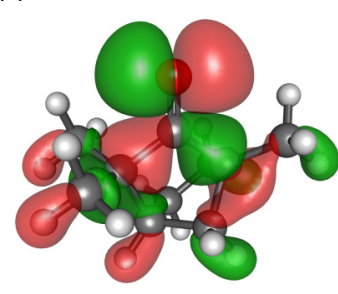

FIG. 1. (Color online) HOMO orbitals of the molecules camphor (a) and fenchone (b) as obtained from GAUSSIAN [32]. The CO bond points upwards.

\section{PECD WITH SCATTERING WAVES}

To overcome the limitations inherent in the SFA, we replace the plane-wave continuum state in Eq. (1) with the Born approximation,

$\psi_{\mathbf{k}}^{(-)}(\mathbf{r})=\frac{1}{(2 \pi)^{3 / 2}}\left[e^{i \mathbf{k r}}-\frac{1}{2 \pi} \int d^{3} r^{\prime} \frac{e^{-i k\left|\mathbf{r}-\mathbf{r}^{\prime}\right|}}{\left|\mathbf{r}-\mathbf{r}^{\prime}\right|} V\left(\mathbf{r}^{\prime}\right) e^{i \mathbf{k r} \mathbf{r}^{\prime}}\right]$,

known from scattering theory. We use the same Volkov phase factor $e^{i S(t)}$ as in the plane-wave SFA, although it is not strictly valid for our corrected continuum states. This leads us to the following continuum-state-corrected SFA (ccSFA) as a replacement for the SFA momentum distribution in Eq. (1):

$$
w^{\mathrm{ccSFA}}(\mathbf{k})=\int d^{3} \mathcal{R}\left|-i \int_{-\infty}^{\infty} d t\left\langle\psi_{\mathbf{k}+\mathbf{A}(t)}^{(-) \mathcal{R}}|\mathbf{r} \cdot \mathbf{E}(t)| \psi_{0}^{\mathcal{R}}\right\rangle e^{i S(t)}\right|^{2}
$$

Note that the continuum state $\psi_{\mathbf{k}+\mathbf{A}(t)}^{(-) \mathcal{R}}$ is now orientation dependent, since the potential $V$ depends on the molecular orientation $\mathcal{R}$. As the initial orbital $\psi_{0}^{\mathcal{R}}$ for our numerical computations, we use the highest occupied molecular orbital (HOMO) obtained with the quantum chemistry software GAUSSIAN [32] using the Becke three-parameter Lee-YangParr (B3LYP) method $[33,34]$ and a $6-31+\mathrm{G}^{*}$ basis set [see Figs. 1(a) and 1(b)]. The potential $V$ in Eq. (7) is the KohnSham potential using the electron density from GAUSSIAN. The integral in Eq. (7) converges for all $\mathbf{k}$ if $r^{2} V(\mathbf{r}) \rightarrow 0$ for $r \rightarrow \infty$. This is true for most practically used Kohn-Sham potentials. For the PECD effect, we conjecture that only the short-range part of $V$ is important, since it is this part that carries signatures of the chiral structure of the molecule. For the results shown in this paper, we use the exchange-only local density approximation (LDA) functional to determine the potential $V$. We have also performed calculations with the full LDA functional and a generalized gradient functional [35] computed with LIBXC [36]. However, the choice of exchangecorrelation potential has only little influence on the results. The values for $I_{p}$ required in Eq. (8) via $S(t)$ for camphor $(8.7 \mathrm{eV})$ and fenchone $(8.6 \mathrm{eV})$ are taken from the literature [22,37].

Figure 2 illustrates exemplary results for the PECD of (R)camphor irradiated with a left-circularly polarized (LCP) 20cycle $\sin ^{2}$-shaped pulse at a wavelength of $398 \mathrm{~nm}$ and an intensity of $2.5 \times 10^{13} \mathrm{~W} / \mathrm{cm}^{2}$. The polarization is defined as

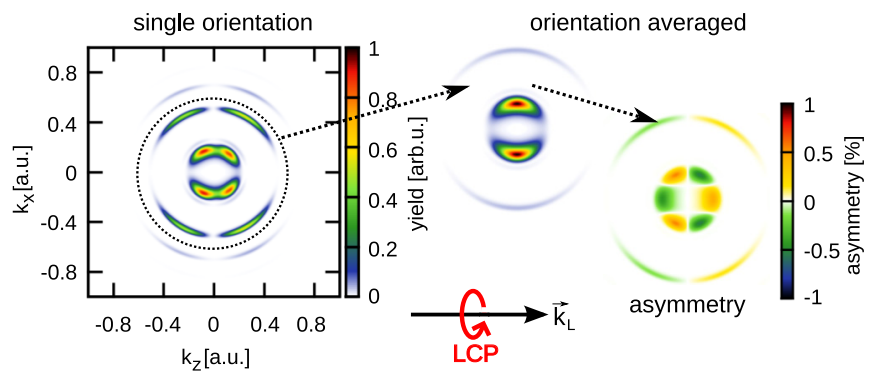

FIG. 2. (Color online) Illustration of the PECD effect in camphor. On the left, a cut $\left(k_{y}=0\right)$ through a momentum distribution at an arbitrary orientation of camphor is shown. In the middle, the corresponding orientation-averaged distribution is shown, and on the right the difference between forward and backward emission is shown in units relative to the average amplitude on the inner (three-photon) ring. The LCP [as defined in Eq. (9)] laser pulse propagates from left to right, as indicated by the arrow.

from the point of view of the receiver, i.e.,

$$
\mathbf{E}_{\mathrm{LCP}}(t)=E_{0}(t)(\cos \omega t, \sin \omega t, 0)
$$

Here, we use 100 points to sample $\xi$ and 80 points for $\eta$ and $\chi$, respectively. To overcome the ionization potential of camphor, absorption of three photons is required. The resulting forward-backward asymmetry takes a maximum value of only about $\pm 1 \%$ relative to the average yield of the momentum distribution on the three-photon ring compared to the $\approx \pm 10 \%$ found in Refs. [26,27]. The nodal structure is in agreement with the results found experimentally [26], although the overall PECD is of opposite sign. The calculated PECD image for (R)-fenchone (not shown) for the given laser parameters yields a slightly stronger PECD effect in our calculation and has no nodes within the forward and backward hemispheres. This is in agreement with the experiment, which found a stronger PECD effect in fenchone molecules as well [26].

In order to investigate the influence of the absorbed photons above threshold, we calculate the PECD for camphor and fenchone for three-, four-, and five-photon ionization. In order to make these calculations numerically more efficient we use here and in all following results the same laser frequency and intensity as stated above, but now as a cw laser field with $E_{0}(t)=$ const. The $\mathrm{cw}$ field enforces exact invariance of the orientation-averaged momentum distribution under rotation about the field propagation axis, i.e., the distribution is cylindrically symmetric and independent of the azimuthal angle $\phi$. In Eq. (8), we make use of the time-translation symmetry with respect to the period $T=2 \pi / \omega$ of the $\mathrm{cw}$ field. Using this symmetry, Eq. (8) takes the simpler form [5]

$$
\begin{aligned}
& w_{\mathrm{cw}}^{\mathrm{ccSFA}}(\mathbf{k})=\omega^{2} \sum_{N=1}^{\infty} \delta^{2}\left(\frac{k^{2}}{2}+I_{p}+U_{p}-N \omega\right) w_{N}(\theta) \\
& w_{N}(\theta)=\int d^{3} \mathcal{R}\left|-i \int_{0}^{T} d t\left\langle\psi_{k_{N} \hat{\mathbf{k}}+\mathbf{A}(t)}^{(-) \mathcal{R}}|\mathbf{r} \cdot \mathbf{E}(t)| \psi_{0}^{\mathcal{R}}\right\rangle e^{i S(t)}\right|^{2}
\end{aligned}
$$

with $U_{p}=E_{0}^{2} / 2 \omega^{2}$ being the ponderomotive potential for circular polarization and $N$ the number of absorbed photons. 
(a) camphor

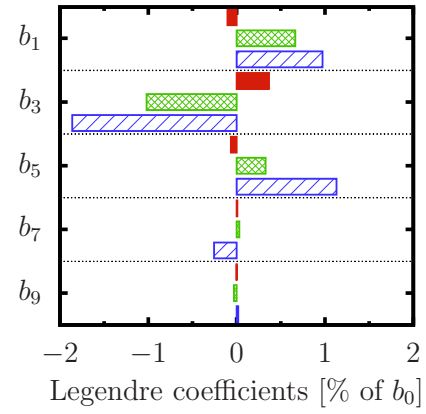

(b) fenchone

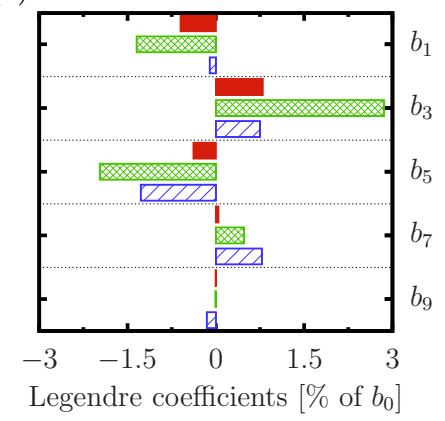

FIG. 3. (Color online) Legendre coefficients $b_{2 n+1}$ for the molecules (a) camphor and (b) fenchone according to Eq. (12). Shown are the values for three-photon ionization (red, solid), four-photon ionization (green, crossed), and five-photon ionization (blue, striped), which are normalized such that the coefficient $b_{0}$ (the total ionization yield) is one for each case. A LCP cw field of $398 \mathrm{~nm}$ wavelength and an intensity of $2.5 \times 10^{13} \mathrm{~W} / \mathrm{cm}^{2}$ have been used.

The vector $\hat{\mathbf{k}}=\mathbf{k} / k_{N}$ is the unit vector pointing in the direction of $\mathbf{k}$ given by its spherical coordinates $(\theta, \phi)$ with $\theta$ being the angle between the laser propagation axis and the velocity of the photoelectron and $\phi$ being the azimuth. $k_{N}$ is the absolute value of momentum corresponding to the absorption of $N$ photons. The reduced momentum distributions $w_{N}(\theta)$ can be decomposed into series of Legendre polynomials $P_{j}(\cos \theta)$ :

$$
w_{N}(\theta)=\sum_{j=0}^{\infty} b_{j, N} P_{j}(\cos \theta) .
$$

In perturbation theory, the highest contributing Legendre polynomial is of order $j_{\max }=2 N$ [8]. The decomposition of the PADs of camphor and fenchone for three-, four-, and five-photon ionization and for a LCP cw field is shown in Fig. 3. For every photon number $N$, the PECD effect can be cast into a single number $\mathcal{A}_{N}$ describing twice the difference between forward and backward emission of photoelectrons relative to the yield per hemisphere [38]. By integration of Eq. (12) over the forward and the backward hemisphere, one obtains

$$
\mathcal{A}_{N}=2 b_{1, N}-\frac{1}{2} b_{3, N}+\frac{1}{4} b_{5, N}-\frac{5}{32} b_{7, N}+\frac{7}{64} b_{9, N}-\cdots .
$$

The even polynomials do not contribute to $\mathcal{A}_{N}$, since they are symmetric with respect to $\theta \rightarrow \pi-\theta$. Thus only the coefficients of odd polynomials are shown in Fig. 3. We observe that for an increasing number of photons, also the

TABLE I. Total PECD $\mathcal{A}_{N}$ according to Eq. (13) for the data shown in Fig. 3 for $N$-photon ionization of camphor and fenchone with the normalization $b_{0}=1$.

\begin{tabular}{lrr}
\hline \hline$N$ & Camphor & Fenchone \\
\hline 3 & $-0.41 \%$ & $-1.74 \%$ \\
4 & $1.92 \%$ & $-4.71 \%$ \\
5 & $2.91 \%$ & $-0.74 \%$ \\
\hline \hline
\end{tabular}

number of contributing Legendre polynomials increases, as predicted by perturbation theory. At the same time, the strength of the PECD increases for camphor, while for fenchone the maximum PECD is obtained for four-photon ionization (see Table I). Comparing our results with the experimental findings [26,27] for three-photon ionization, we conclude that quantitative agreement is not achieved, since the measured asymmetries of about $10 \%$ are not reproduced. However, qualitative agreement is achieved in the sense that the third coefficient dominates the distribution for camphor but not for fenchone. Experimentally, the opposite direction of asymmetry has been found for three-photon ionization. The fact that camphor and fenchone exhibit the same sign of asymmetry for three-photon ionization is in accordance with the experiments.

\section{INFLUENCE OF INDIVIDUAL MOLECULAR ORIENTATIONS ON THE PECD EFFECT}

In order to investigate the origin of the PECD effect, we study the contributions of individual molecular orientations.

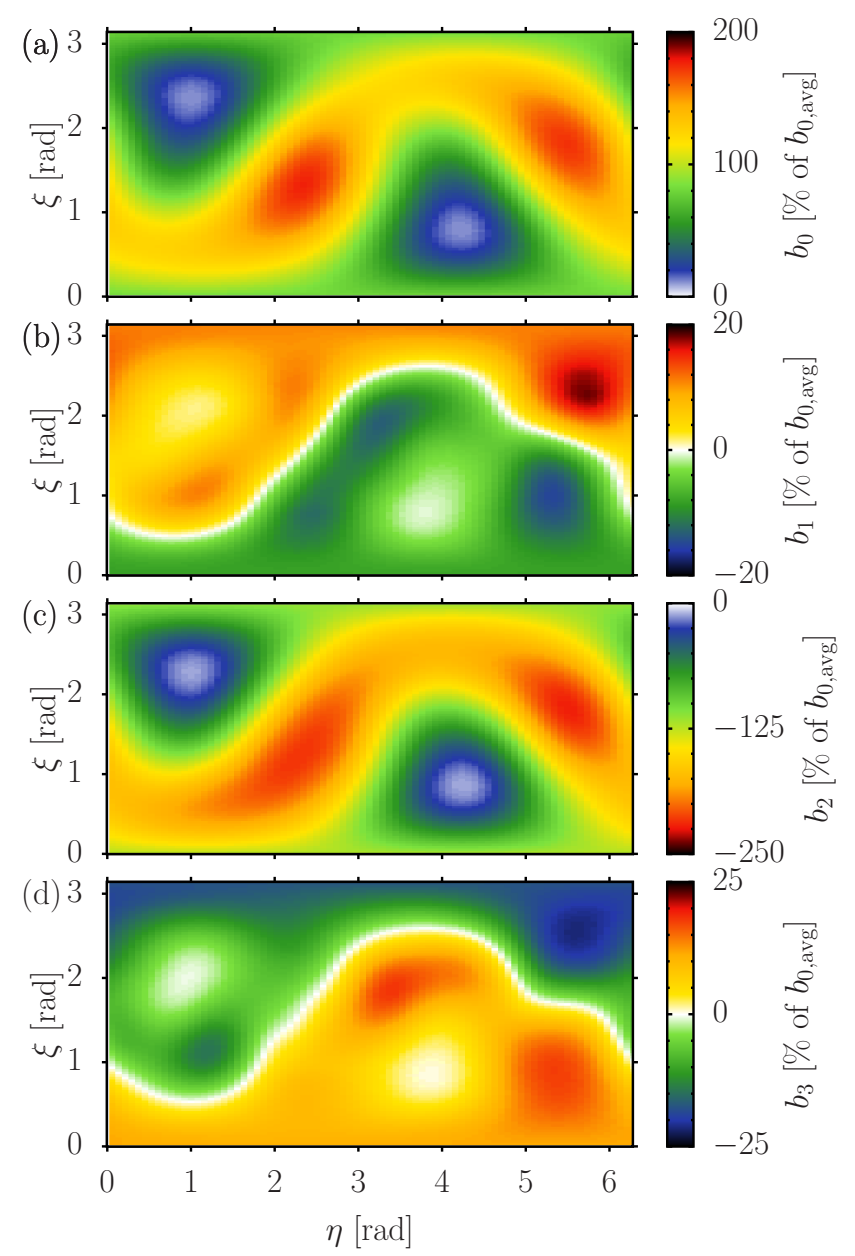

FIG. 4. (Color online) Legendre coefficients $b_{0}$ (a), $b_{1}$ (b), $b_{2}$ (c), and $b_{3}$ (d) for camphor in dependence of the molecular orientation $(\eta, \xi)$. All values are given relative to the average yield $b_{0, \text { avg }}$ and correspond to three-photon ionization. Note the different color scales in the plots. 


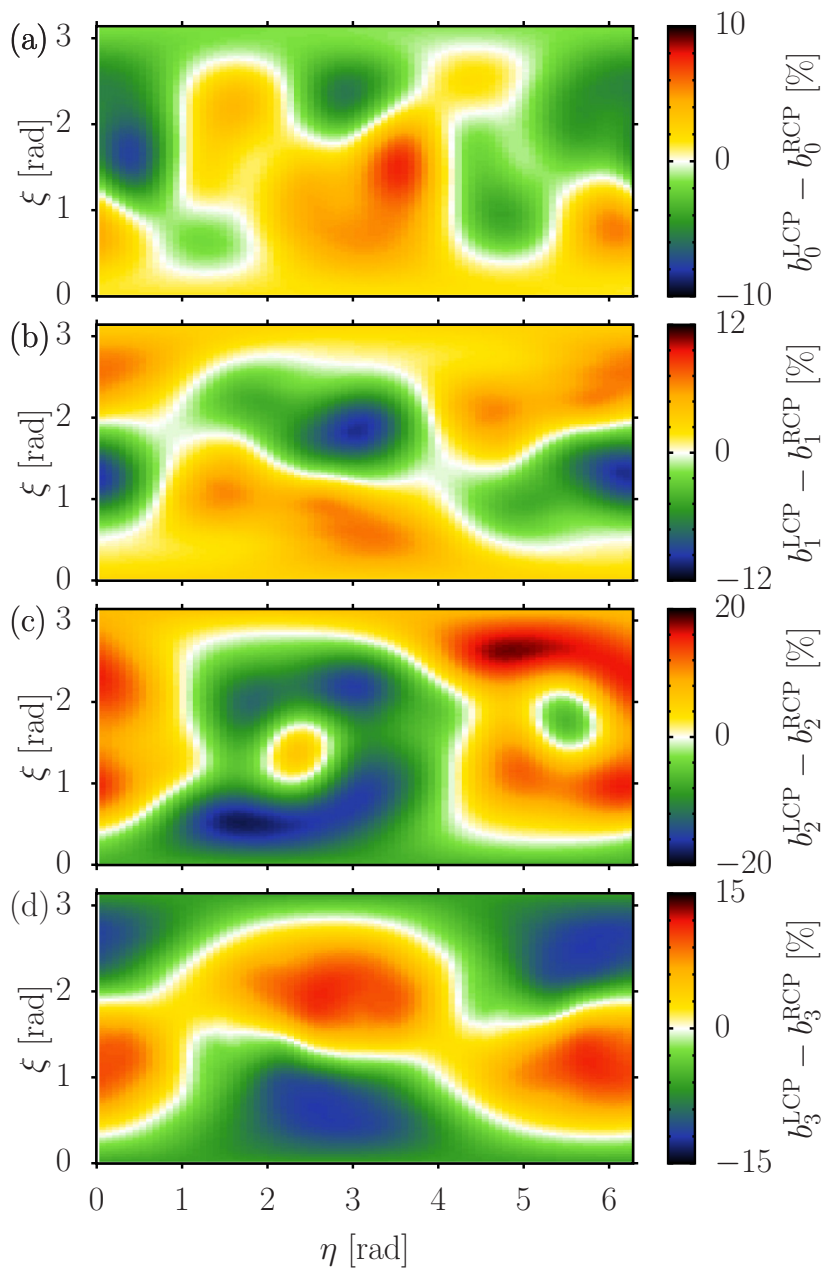

FIG. 5. (Color online) Dichroism of each molecular orientation $(\eta, \xi)$ of camphor, decomposed into the Legendre coefficients (a) $b_{0}$, (b) $b_{1}$, (c) $b_{2}$, and (d) $b_{3}$. All values are given in relation to the average yield $b_{0 \text {,avg }}$ and correspond to three-photon ionization. Note the different color scales in the plots.

This may help to understand the process more intuitively. Here, we consider only three-photon transitions in camphor and fenchone, for the same parameters as given in the previous section. The relevant molecular orientations are given by the Euler angles $\eta$ and $\xi$, which rotate the molecule around the $z$ axis (laser propagation axis) and the $y$ axis consecutively. We integrate over the third Euler angle $\chi$, so that the resulting distribution is independent of the azimuthal angle $\phi$ and decomposition into Legendre polynomials is possible for each orientation $(\eta, \xi)$. The initial orientation $\eta=\xi=0$ is chosen for both camphor and fenchone such that the $\mathrm{CO}$ bond points in the $z$ direction. The first Legendre coefficients $b_{0}, b_{1}, b_{2}$, and $b_{3}$ are shown for camphor as a function of $\eta$ and $\xi$ in Fig. 4. All values are normalized to the average value $b_{0 \text {,avg }}$ of $b_{0}$. Note that the zero-order Legendre coefficient $b_{0}$ corresponds to the ionization yield in the three photon channel. Not surprisingly, the angular momentum distribution-and thus the Legendre coefficients-depend strongly on the molecular orientation. From Eqs. (11) and (12) it is obvious that the Legendre coefficients for a sample of randomly oriented molecules are just integrals of the Legendre coefficients for individual

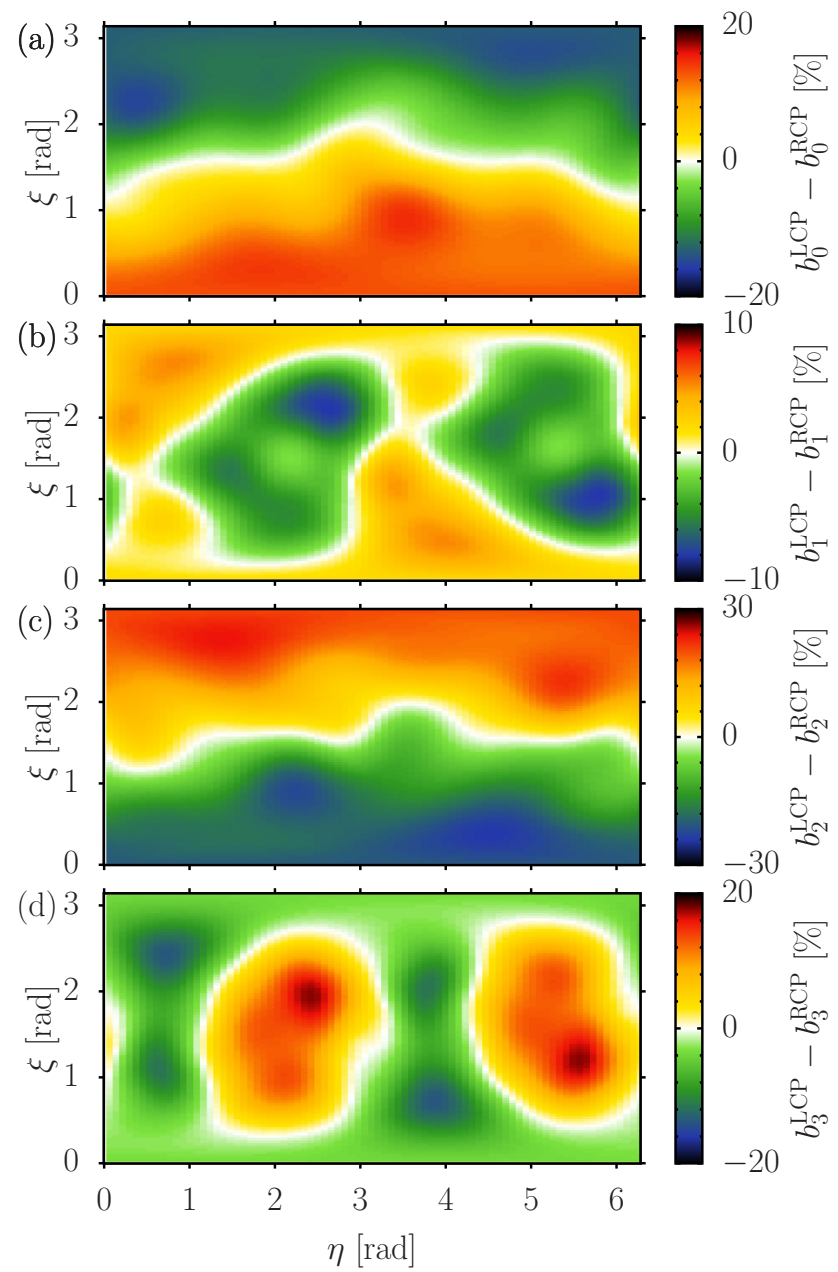

FIG. 6. (Color online) Same as Fig. 5, but for fenchone.

orientations with respect to the Euler angles $\eta$ and $\xi$. Hence, the large positive and negative Legendre coefficients in Figs. 4(b) and 4(d) almost cancel each other. We can investigate the dichroism of individual orientations $(\eta, \xi)$ by computing the difference between the Legendre coefficients for LCP and for right-circularly polarized (RCP) light for each molecular orientation. The results are shown in Figs. 5 and 6 for camphor and fenchone, respectively. From these figures, we observe that the individual molecular orientations show a very pronounced dichroism in the even as well as in the odd coefficients. The (anti)symmetry visible in these pictures, is due to the following (anti)symmetries in the Legendre coefficients:

$$
\begin{gathered}
b_{2 j}^{\mathrm{LCP}}(\eta, \xi)=b_{2 j}^{\mathrm{RCP}}(\eta+\pi, \pi-\xi), \\
b_{2 j+1}^{\mathrm{LCP}}(\eta, \xi)=-b_{2 j+1}^{\mathrm{RCP}}(\eta+\pi, \pi-\xi) .
\end{gathered}
$$

These relations can be easily verified using the time-dependent Schrödinger equation in the dipole approximation. Hence, the dichroism in the even coefficients vanishes already when we sum each two opposing orientations $(\eta, \xi)$ and $(\eta+\pi, \pi-\xi)$. Although this is not true for the odd coefficients, we see areas with positive and negative dichroism that cancel each other to some extent. By this, the dichroism of the oriented molecules can easily reach $20 \%$, whereas the total PECD 
as computed in the previous section amounts to only a few percent. This in turn means that the PECD effect of a gas of molecules will be strongly dependent on any form of molecular alignment such as orientation-dependent resonant enhancement of ionization [27], which may be a reason for the large quantitative disagreement between our numerical results and the measured PECD [26,27].

\section{CONCLUSIONS}

We have shown a possibility to calculate the PECD of chiral molecules nonperturbatively. The commonly used SFA fails in describing this effect, which leads to the conclusion that not only the orbital from which ionization takes place is important, but also the final scattering state of the emitted electron. This is in accordance with findings in the single-photon PECD, where an asymmetry occurs even with an achiral initial orbital, indicating that the scattering state plays a vital role in the process $[39,40]$. We have demonstrated that a rather simple approximation for the scattering state is sufficient to yield a nonzero PECD effect and thus to overcome a fundamental limitation of the plain SFA. However, quantitative agreement with the experimental data is not achieved. This may be due to the Born approximation, which is only a first-order correction. Additionally, we have neglected the influence of resonant intermediate states that may influence the process, especially if the resonance is orientation dependent. We have shown that individual orientations exhibit a very strong dichroism that is canceled to some extent by orientation averaging. This suggests that any form of effective molecular alignment could lead to a significant change in the total PECD. This will be the subject of further research.

\section{ACKNOWLEDGMENT}

We thank the Deutsche Forschungsgemeinschaft for funding the Centre for Quantum Engineering and Space-Time Research (QUEST).
[1] L. V. Keldysh, Sov. Phys. JETP 20, 1307 (1965).

[2] F. H. M. Faisal, J. Phys. B 6, L89 (1973).

[3] H. R. Reiss, Phys. Rev. A 22, 1786 (1980).

[4] M. Lewenstein, P. Balcou, M. Y. Ivanov, A. L'Huillier, and P. B. Corkum, Phys. Rev. A 49, 2117 (1994).

[5] A. Becker and F. H. M. Faisal, J. Phys. B: At. Mol. Opt. Phys. 38, R1 (2005).

[6] C. I. Blaga, F. Catoire, P. Colosimo, G. G. Paulus, H. G. Muller, P. Agostini, and L. F. DiMauro, Nat. Phys. 5, 335 (2008).

[7] D. Shafir, H. Soifer, B. D. Bruner, M. Dagan, Y. Mairesse, S. Patchkovskii, M. Y. Ivanov, O. Smirnova, and N. Dudovich, Nature (London) 485, 343 (2012).

[8] K. L. Reid, Mol. Phys. 110, 131 (2012).

[9] T. K. Kjeldsen, C. Z. Bisgaard, L. B. Madsen, and H. Stapelfeldt, Phys. Rev. A 71, 013418 (2005).

[10] E. Hasović and D. B. Milošević, Phys. Rev. A 86, 043429 (2012).

[11] A. Jaroń-Becker and A. Becker, Laser Phys. 19, 1705 (2009).

[12] A. T. J. B. Eppink and D. H. Parker, Rev. Sci. Instrum. 68, 3477 (1997).

[13] M. Wollenhaupt, M. Krug, J. Köhler, T. Bayer, C. SarpeTudoran, and T. Baumert, Appl. Phys. B 95, 647 (2009).

[14] J. L. Hansen, H. Stapelfeldt, D. Dimitrovski, M. Abu-samha, C. P. J. Martiny, and L. B. Madsen, Phys. Rev. Lett. 106, 073001 (2011).

[15] E. Shigemasa, J. Adachi, M. Oura, and A. Yagishita, Phys. Rev. Lett. 74, 359 (1995).

[16] O. Gessner, A. M. D. Lee, J. P. Schaffer, H. Reisler, S. V. Levchenko, A. I. Krylov, J. G. Underwood, H. Shi, A. L. L. East, D. M. Wardlaw, E. t. H. Chrysostom, C. C. Hayden, and A. Stolow, Science 311, 219 (2006).

[17] L. Holmegaard, J. L. Hansen, L. Kalhøj, S. L. Kragh, H. Stapelfeldt, F. Filsinger, J. Küpper, G. Meijer, D. Dimitrovski, M. Abu-samha, C. P. J. Martiny, and L. B. Madsen, Nat. Phys. 6, 428 (2010).

[18] B. Ritchie, Phys. Rev. A 13, 1411 (1976).

[19] N. A. Cherepkov, Chem. Phys. Lett. 87, 344 (1982).

[20] L. Nahon, G. A. Garcia, C. J. Harding, E. Mikajlo, and I. Powis, J. Chem. Phys. 125, 114309 (2006).
[21] N. Böwering, T. Lischke, B. Schmidtke, N. Müller, T. Khalil, and U. Heinzmann, Phys. Rev. Lett. 86, 1187 (2001).

[22] I. Powis, C. J. Harding, G. A. Garcia, and L. Nahon, Chem. Phys. Chem. 9, 475 (2008).

[23] D. Catone, M. Stener, P. Decleva, G. Contini, N. Zema, T. Prosperi, V. Feyer, K. C. Prince, and S. Turchini, Phys. Rev. Lett. 108, 083001 (2012).

[24] U. J. Meierhenrich, Amino Acids and the Asymmetry of Life (Springer, Berlin, 2008).

[25] L. Nahon, G. Garcia, I. Powis, U. Meierhenrich, and A. Brack, Proc. SPIE 6694, 669403 (2007).

[26] C. Lux, M. Wollenhaupt, T. Bolze, Q. Liang, J. Köhler, C. Sarpe, and T. Baumert, Angew. Chem. Int. Ed. 51, 5001 (2012).

[27] C. S. Lehmann, N. B. Ram, I. Powis, and M. H. M. Janssen, J. Chem. Phys. 139, 234307 (2013).

[28] I. Powis, J. Chem. Phys. 112, 301 (2000).

[29] M. Stener, D. D. Tommaso, G. Fronzoni, P. Decleva, and I. Powis, J. Chem. Phys. 124, 024326 (2006).

[30] M. A. Morrison and G. A. Parker, Aust. J. Phys. 40, 465 (1987).

[31] E. P. Wigner, Group Theory and its Application to the Q.M. of Atomic Spectra (Academic, New York, 1959).

[32] M. J. Frisch et al., Gaussian 09, revision c.01 (Gaussian, Inc., Wallingford CT, 2010).

[33] C. Lee, W. Yang, and R. G. Parr, Phys. Rev. B 37, 785 (1988).

[34] A. D. Becke, J. Chem. Phys. 98, 5648 (1993).

[35] A. D. Boese and N. C. Handy, J. Chem. Phys. 114, 5497 (2001).

[36] M. A. L. Marques, M. J. T. Oliveira, and T. Burnus, Comput. Phys. Commun. 183, 2272 (2012).

[37] E. E. Rennie, I. Powis, U. Hergenhahn, O. Kugeler, G. Garcia, T. Lischke, and S. Marburger, J. Electron Spectrosc. Relat. Phenom. 125, 194 (2002).

[38] M. H. M. Janssen and I. Powis, Phys. Chem. Chem. Phys. 16, 856 (2014).

[39] C. J. Harding, E. Mikajlo, I. Powis, S. Barth, S. Joshi, V. Ulrich, and U. Hergenhahn, J. Chem. Phys. 123, 234310 (2005).

[40] I. Powis, Chirality 20, 961 (2008). 